\title{
RENDIMENTO E COMPOSIÇÃO QUÍMICA DE CULTIVARES DE MILHO EM MOAGEM A SECO E PRODUÇÃO DE GRITS
}

\author{
ROGÉRIO AMARO GONÇALVES ${ }^{1}$ \\ JAMILTON PEREIRA DOS SANTOS ${ }^{2}$ \\ PEDRO HENRIQUE FERREIRA TOMÉ ${ }^{1}$ \\ ROSEMARY G. F. A. PEREIRA ${ }^{3}$ \\ JOSÉ LUIZ RAMIREZ ASCHERI ${ }^{4}$ \\ CELESTE MARIA PATTO DE ABREU
}

RESUMO - O milho é uma das principais matérias-
primas para a indústria de alimentos. Desse cereal, é
possível obter óleo, fubá, canjica, grits, farelo, amido e
zeína. Conduziu-se este trabalho com o objetivo de ava-
liar o rendimento de moagem a seco, produção de grits
e composição química de 16 cultivares de milho. Os
grãos de milho das 16 cultivares avaliadas tiveram a
sua umidade padronizada para $12 \%$ e foram submetidos
a um processo de limpeza em uma mesa gravimétrica.
Encerrada a etapa de limpeza, foram colocados 10 kg
de grãos de cada cultivar em degerminador de facas
com rotação de 600 rpm por um período de 10 minutos.
Após a degerminação, o endosperma foi passado por
moinho de rolos corrugados com distância entre rolos
de $30 \mu$ m. O material moído foi submetido à tamisação
em peneiras de $14,20,25$ e 30 mesh por um período de

10 minutos. Para a degerminação, o delineamento experimental utilizado foi o inteiramente casualizado, com um fator de 16 cultivares e 2 repetições. Na tamisação, o delineamento usado foi com dois fatores: 16 cultivares, 5 peneiras e 2 repetições. A cultivar BR106, o híbrido duplo BRS-2114 e o híbrido triplo BR3123 registraram maiores rendimentos $(\mathrm{P} \leq 0,05)$ na fração endosperma. Na tamisação, a fração avaliada foi a retida em peneira de 20 mesh, por causa da utilização de grits com essas dimensões como matéria-prima na indústria de "snacks". Os híbridos triplos BR-3123, BRS-3101 e BR 106 foram os mais produtivos na peneira de 20 mesh, sendo os mais indicados para a indústria produtora de "grits", fornecedora de matériaprima para a indústria de alimentos, entre essas, as de "snacks"e a de bebidas.

TERMOS PARA INDEXAÇÃO: Milho, moagem, degerminação, processamento, rendimento, Zea mays.

\section{YIELD AND CHEMICAL COMPOSITION OF CORN CULTIVATE IN DRY-MILLING AND GRITS PRODUCTION}

\begin{abstract}
Maize is one of the major source of energetic an nutritious ingredient for the food industry. From the maize grain it is possible to get oil, meal, grits, bran, starch, zein. The objectives of this research was to evaluate the dry-miling yield and chemical composition of different experimental maize genotypes. Fourteen genotypes came from the breeding program of Embrapa Corn and Sorghum Researcher Center and two came from commercial companies. The grain
\end{abstract}

moisture content of all cultivars were equilibrated at $12 \%$ and dust was removed in a gravimetric board, before processing. Grain samples of each type was placed in a blade degermer at $600 \mathrm{rpm}$ rotation speed for 10 minutes. The endosperm portion, after degermination, was transferred to a mill roll. Following the milling process grounded material was sifted in 14, 20, 25 and 30 mesh sifters for 10 minutes. For the degermination the used experimental design was

\footnotetext{
1. Doutor em Ciência dos Alimentos.

2. Pesquisador III - Embrapa Milho e Sorgo.

3. Docente Departamento Ciência dos Alimentos da UNIVERSIDADE FEDERAL DE LAVRAS/UFLA, Caixa Postal 37 - 37200-000 - Lavras, MG.

4. Docente Departamento de Química/UFLA.

5. Pesquisador III - Embrapa Agroindústria de Alimentos.
} 
randomized entirely with a factor 16 cultivate and two repetitions. In the sifting the used design was with two factors: 16 cultivate, five sieves and two repetitions. The BR 106, BRS 2114, BR 3123, Bozeme Amarelo,
BRS 3101, BR 206, HD-951128, BRS 4150 and BR 205 showed higher endosperm yield. The triple hybrids BR 3123, BRS 3101 and the variety BR 106 showed better characteristics for a grits production in the 20 mesh sifter.

INDEX TERMS: Maize, milling, degermination, processing, yield, Zea mays.

\section{INTRODUÇÃO}

A transformação do milho em diversos derivados possibilita o uso desse cereal como excelente fonte de matéria-prima para a indústria de alimentos.

Do milho, obtêm-se em torno de noventa derivados diferentes; entre esses, os principais são grits, fubá, canjica, óleo, amido, amilose, amilopectina, zeína e fibras.

Uma das principais aplicações do milho na indústria de alimentos é o uso do grão degerminado e moído para a produção de "fast food" em forma de alimentos extrudados conhecidos como "snacks".

A caracterização de um milho como superprecoce, precoce e normal é função da emissão da inflorescência. $\mathrm{O}$ milho superprecoce é aquele que emite a inflorescência 50 dias após o plantio. A cultivar definida como precoce emite a inflorescência 55 a 65 dias após a semeadura, e o milho caracterizado como normal emite a inflorescência após 65 dias do plantio. Essa caracterização varia de uma região para outra, em função do grau de insolação incidente sobre o milho.

O grão degerminado e moído, que neste trabalho chama-se grits de milho, apresenta em sua composição em torno de $90 \%$ de amido. Esse composto é importante fonte de matéria-prima na indústria de alimentos, bebidas e em extrusão termoplástica.

A avaliação do rendimento de moagem em milho após a degerminação é um parâmetro importante para a indústria de alimentos e para os melhoristas.

Brekke (1967) verificou que o précondicionamento com $15 \%$ de umidade por um período de 20 horas aumentou o rendimento das diversas frações de grits quando se utiliza um degerminador Bell.

Baixo teor de cinzas em grãos de milho dá alto rendimento de moagem, produzindo uma fração de semolina com baixo teor de lipídio (MESTRES et al., 1991).

Milhos com altos teores de lisina produzem baixo rendimento nas frações de grits quando moídos a seco, devido à deficiência desses grãos em endosperma córneo (BREKKE et al., 1971).
Peplinski et al. (1984) avaliaram o rendimento de moagem de milho com condicionamento com teores de umidade mais baixos e menores tempos de condicionamento e obtiveram grits com baixo teor de lipídios.

Conduziu-se este trabalho com o objetivo de avaliar diferentes cultivares de milho no processo de moagem a seco e produção de grits retidos em peneira de 20 mesh.

\section{MATERIAL E MÉTODOS}

\section{Matéria-prima}

Foram utilizadas 16 cultivares de milho da safra 2000 cultivadas na Estação Experimental da Embrapa Milho e Sorgo, com as características apresentadas na Tabela 1.

\section{Processamento do milho}

O processamento do milho foi realizado por via seca, com os grãos fluindo para um secador FabbePrimar, onde atingiram a umidade de $12 \%$, passando por mesa gravimétrica; em seguida, foram conduzidos $10 \mathrm{~kg}$ de grãos de milho de cada repetição das cultivares estudadas a um degerminador com rotação do eixo das facas de $600 \mathrm{rpm}$, onde foi separado e pesado o endosperma do germe. O tempo de degerminação estabelecido foi de 10 minutos (Figura 1).

Após a degerminação, o grão degerminado foi moído em um moinho de rolo corrugado, modelo Tecmolin, com distância entre rolos de $30 \mu \mathrm{m}$. O material moído foi peneirado por 5 minutos em um aparelho RL - 10, utilizando peneiras de 14, 20, 25, 30 mesh e fundo; em seguida, foram pesadas as frações retidas em cada peneira.

O material retido em peneira de 20 mesh, neste trabalho chamado de grits, foi definido como produto obtido pela moagem do grão de milho degerminado com granulometria na faixa de 590 a $1680 \mu \mathrm{m}$.

Na Figura 2 encontra-se o fluxograma do processamento de milho.

Ciênc. agrotec., Lavras. V.27, n.3, p.643-650, maio/jun., 2003 
TABELA 1 - Cultivares de milho utilizadas na avaliação de rendimento em moagem a seco.

\begin{tabular}{ccc}
\hline Nome & Característica & Formato do Grão \\
HD 951128 & Superprecoce & Dentado \\
HT 971011 & Precoce & Duro \\
BRS 3133 & Superprecoce & Duro \\
HT $7105-3$ & Precoce & Semiduro \\
BRS 3150 & Precoce & Semidentado \\
Bozeme Amarelo & Precoce & Duro \\
BRS 2114 & Superprecoce & Semidentado \\
BRS 3101 & Precoce & Semiduro \\
BR 205 & Precoce & Semidentado \\
BRS 4150 & Normal & Semidentado \\
BR 206 & Precoce & Semidentado \\
BRS 3060 & Normal & Semiduro \\
BR 106 & Normal & Semidentado \\
BR 3123 & Superprecoce & Semiduro \\
HS - TR 3 & Superprecoce & Semiduro \\
D - 1000 & Precoce &
\end{tabular}

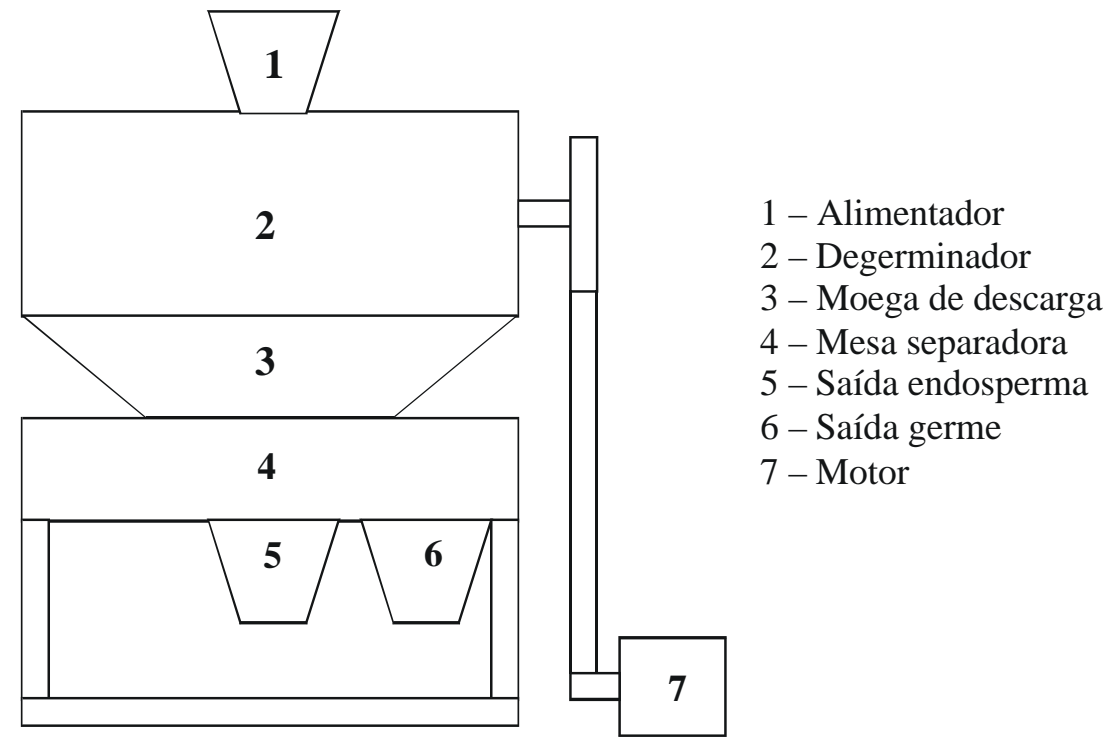

FIGURA 1 - Degerminador de facas. 


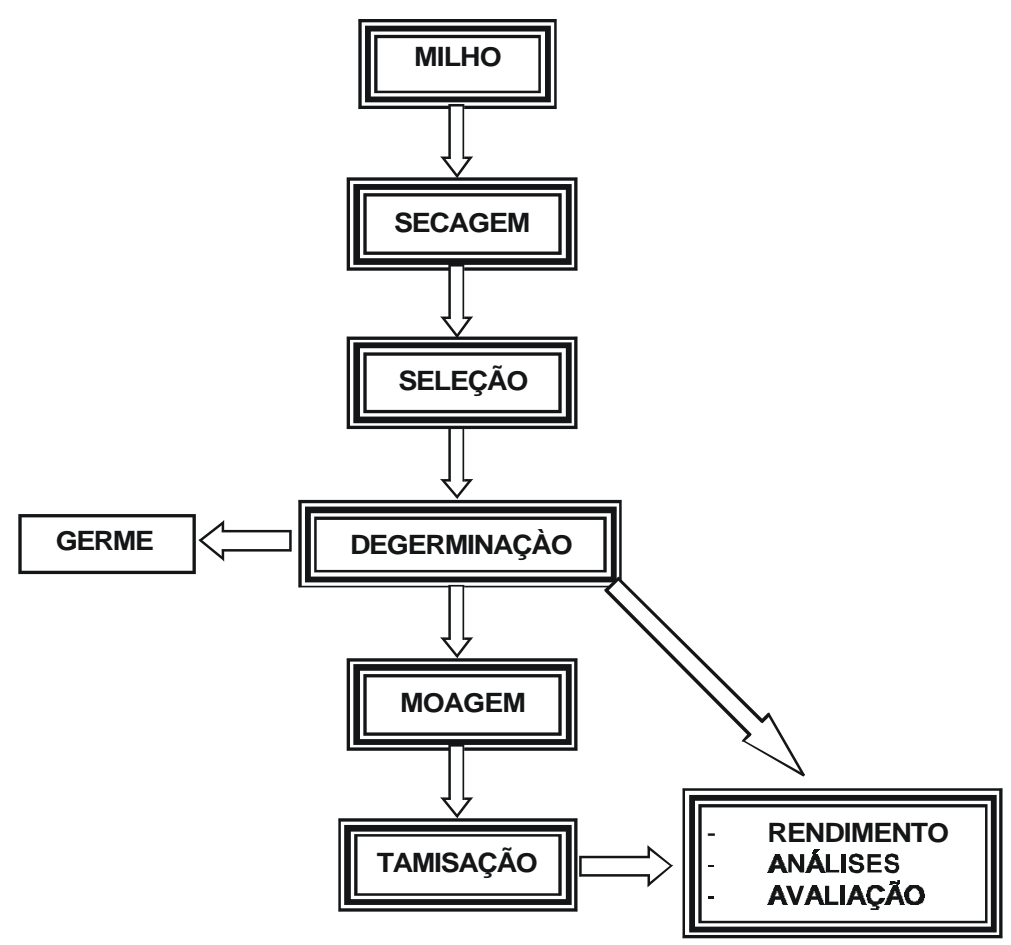

FIGURA 2 - Fluxograma do processamento de milho via seca.

Foi usado o delineamento experimental inteiramente casualizado (dic) contando 16 tratamentos (cultivares) e duas repetições apresentando um total de 32 parcelas experimentais (tabela 2). Para a avaliação da tamisação, foi acrescentado o fator peneiras (5 níveis: 14, 20, 25 e 30 mesh, e fundo).

\section{Análises}

O rendimento de endosperma, germe e grits foi realizado por meio de pesagens e comparando-se o resultado com o peso da amostra de grãos de milho no caso do germe e endosperma, e para os grits, a comparação foi feita somente com a fração endosperma.

A umidade foi determinada de acordo com o método $\mathrm{n}^{\mathrm{o}}$ 44-15A (AACC, 1983), colocando-se $10 \mathrm{~g}$ de amostra em estufa com circulação de ar a $130^{\circ} \mathrm{C}$ por 1 hora.

A proteína foi determinada obedecendo ao método $\mathrm{n}^{\mathrm{o}}$ 46-13 (AACC, 1983), método microKjeldahl. Para a determinação de lipídios, o método utilizado foi o n⿳- 30-25 (AACC, 1983), utilizando extrator Soxhlet. As cinzas foram analisadas segundo o método $\mathrm{n}^{\mathrm{o}}$ 08-01 (AACC, 1983), mediante calcinação em mufla a $550^{\circ} \mathrm{C}$.

\section{RESULTADOS E DISCUSSÃO}

\section{Degerminação}

Houve diferenças significativas no rendimento de endosperma e germe $(\mathrm{P} \leq 0,05)$ entre as 16 cultivares utilizadas neste trabalho (Tabela 2). Neste trabalho, foi dada ênfase à fração endosperma, em virtude da sua utilização pelos fabricantes de grits.

Observou-se que nove cultivares, sendo oito delas desenvolvidas pela Embrapa Milho e Sorgo (BR 106, BRS 2114, BR 3123, BRS 3101, BR 206, HD951128, BRS 4150 e BR 205) e uma delas comercial (Bozeme Amarelo), destacaram-se pelo maior rendimento na degerminação na fração endosperma.

A indústria processadora de milho via seca necessita de cultivares que tenham maior rendimento de endosperma para a posterior fabricação das diversas frações de grits. O mais baixo desempenho na produção de endosperma foram das cultivares HT-7105-3, BRS 3150, BRS 3133, D-1000, HS-TR-3, BRS 3060 e HT971011. Esses dados estão de acordo com os dados obtidos por Brekke (1967) e Peplinski et al. (1984), que trabalharam com híbridos de diferentes empresas, obtendo grande variabilidade na fração endosperma. 
TABELA 2 - Rendimento $(\mathrm{kg})$ de amostras de $10 \mathrm{~kg}$ de grãos na produção de endosperma e germe em diferentes cultivares de milho.

\begin{tabular}{cccc}
\hline Cultivares & Endosperma* & Germe* & Tipo de Grão \\
\hline BR 106 & $8,85 \mathrm{a}$ & $1,10 \mathrm{~b}$ & Semidentado \\
BRS 2114 & $8,85 \mathrm{a}$ & $1,12 \mathrm{~b}$ & Semidentado \\
BR 3123 & $8,80 \mathrm{a}$ & $1,15 \mathrm{~b}$ & Semiduro \\
Bozeme Amarelo & $8,75 \mathrm{a}$ & $1,20 \mathrm{~b}$ & Duro \\
BRS 3101 & $8,60 \mathrm{a}$ & $1,35 \mathrm{~b}$ & Semiduro \\
BR 206 & $8,60 \mathrm{a}$ & $1,35 \mathrm{~b}$ & Semidentado \\
HD-951128 & $8,60 \mathrm{a}$ & $1,40 \mathrm{a}$ & Dentado \\
BRS 4150 & $8,55 \mathrm{a}$ & $1,40 \mathrm{a}$ & Semidentado \\
BR 205 & $8,55 \mathrm{a}$ & $1,40 \mathrm{a}$ & Semidentado \\
HT-971011 & $8,40 \mathrm{~b}$ & $1,55 \mathrm{a}$ & Duro \\
BRS 3060 & $8,40 \mathrm{~b}$ & $1,55 \mathrm{a}$ & Semiduro \\
HS-TR-3 & $8,35 \mathrm{~b}$ & $1,60 \mathrm{a}$ & Semiduro \\
D-1000 & $8,27 \mathrm{~b}$ & $1,67 \mathrm{a}$ & Duro \\
BRS 3133 & $8,225 \mathrm{~b}$ & $1,77 \mathrm{a}$ & Duro \\
HT-7105-3 & $8,05 \mathrm{~b}$ & $1,95 \mathrm{a}$ & Semiduro \\
BRS 3150 & $8,05 \mathrm{~b}$ & $1,95 \mathrm{a}$ & Semidentado \\
\hline
\end{tabular}

*Médias seguidas de mesma letra nas colunas não diferem $(\mathbf{P} \leq \mathbf{0 , 0 5})$ pelo teste de Scott-Knott (SCOTT e KNOTT, 1974).

\section{Tamisação}

A tamisação foi executada após a passagem do endosperma obtido da degerminação por moinho de cilindros corrugados. Objetivou-se com a tamisação, a avaliação mediante pesagens, da retenção das diferentes frações de grits nas peneiras de 14, 20, 25 e 30 mesh. A indústria processadora de milho fornece às empresas de fabricação de "snacks" grits retidos na peneira de 20 mesh (ROKEY e HUBER, 1992).

A interação cultivares versus peneiras foi estatisticamente significativa $(\mathrm{P}<0,05)$ e o desdobramento cultivares dentro de cada peneira apresentou resultados significativos nas peneiras de 14 e 20 mesh. As amostras retidas nas peneiras de 25 e 30 mesh não apresentaram diferenças significativas. A peneira 20 mesh apresentou maior retenção de grits, dando rendimento de aproximadamente $50 \%$ de todo o material colocado no início da tamisação, e a peneira de 14 mesh reteve em torno de $25 \%$ do total de cada material peneirado (Tabela 3).

Os híbridos triplos BR 3123, BRS 3101 e a cultivar BR 106 semidentada apresentaram maiores valores de grits retidos em peneira de 20 mesh. Sendo, portanto, as mais indicadas para a indústria produtora de grits, fornecedora de matéria-prima para a indústria de "snacks", estando de acordo com os resultados obtidos por Anderson et al. (1969). Esses resultados concordam com os dados obtidos por Brekke (1967), realizados com híbridos de milho. O híbrido HD-951128 apresentou o mais baixo rendimento. As demais cultivares apresentaram o mesmo rendimento de acordo com a análise estatística. 
TABELA 3 - Rendimento $(\mathrm{kg})$ de grits retidos em peneira de 20 mesh.

\begin{tabular}{cc}
\hline Cultivares & Valores Médios (kg)* \\
\hline BR 3123 $3,38 \mathrm{a}$ \\
BRS 3101 & $4,29 \mathrm{a}$ \\
BR 106 & $4,26 \mathrm{a}$ \\
BRS 2114 & $4,16 \mathrm{~b}$ \\
BR 205 & $4,16 \mathrm{~b}$ \\
BR 206 & $4,14 \mathrm{~b}$ \\
Bozeme Amarelo & $4,10 \mathrm{~b}$ \\
BRS 3133 & $4,08 \mathrm{~b}$ \\
BRS 3060 & $4,07 \mathrm{~B}$ \\
BRS 4150 & $4,07 \mathrm{~B}$ \\
D-1000 & $4,06 \mathrm{~B}$ \\
HS-TR-3 & $4,01 \mathrm{~B}$ \\
HT-971011 & $3,98 \mathrm{~B}$ \\
BRS 3150 & $3,95 \mathrm{~B}$ \\
HT-7105-3 & $3,89 \mathrm{~B}$ \\
HD-951128 & $3,55 \mathrm{C}$ \\
\hline
\end{tabular}

*Médias seguidas de mesma letra nas colunas não diferem $(\mathbf{P} \leq \mathbf{0 , 0 5})$ pelo teste de Scott-Knott (SCOTT e KNOTT, 1974).

\section{Análises \\ Proteína}

O resultado das análises do teor de proteína dos grãos das diferentes cultivares avaliadas registrou diferenças significativas, conforme mostrou a análise estatística (Tabela 4). O híbrido simples HSTR-3 registrou maior valor no teor de proteína, isto é, indesejável para fins de produção de grits, visto que, em geral, quanto maior o teor de proteína, menor será o teor de amido, e a fração zeína interfere negativamente na extrusão (ZHANG e HOSENEY, 1998). O híbrido duplo BRS 3123 apresentou o menor valor de proteína e conseqüentemente maior valor de carboidratos. Para utilização da indústria de "snacks", é melhor teor de proteína mais baixo, para garantir maior expansão do "snack" (FLETCHER et al., 1985).

\section{Cinzas}

Também as análises do teor de cinzas mostraram que as cultivares utilizadas neste trabalho apresentaram diferenças significativas $(P \leq 0,05)$, conforme ilustra a Tabela 5. O híbrido simples HS-TR-3 e o híbrido triplo BR 3123 apresentaram maiores valores nos teores de cinza. Para a indústria de "snacks", altos teores de cinzas é indesejável, pois compromete a expansibilidade do produto final. As cultivares BR 206 e HT-7105-3, BRS 3060 e D-1000 registraram menores valores de cinzas. Os dados obtidos estão de acordo com os resultados de pesquisa realizada por Mestres et al. (1991), os quais avaliaram diversas cultivares de milho e verificaram que altos teores de cinzas comprometem o rendimento de grits.

\section{Extrato etéreo}

Os valores de extrato etéreo dos grãos das diferentes cultivares registraram grande variação e apresentaram diferenças significativas, conforme mostrou a análise estatística $(\mathrm{P} \leq 0,05)$ ilustrada na Tabela 4. Os grãos do HT-7105-3 tiveram maiores valores de extrato etéreo. O híbrido triplo BRS 3150 teve o menor valor de extrato etéreo. Para a indústria de grits, quanto menor o valor de extrato etéreo, maior será o rendimento da fração endosperma na degerminação.

\section{Carboidratos}

O teor de carboidratos dos grãos das cultivares utilizadas apresentaram diferenças significativas $(\mathrm{P} \leq 0,05)$, conforme resultados da análise estatística (Tabela 4). Os grãos do BR 3123 tiveram maior valor de carboidratos. Esse parâmetro é importante para a indústria de grits, pois quanto maior o teor de carboidratos, maior será o rendimento na degerminação e tamisação. Os grãos do HS-TR-3 apresentaram menor valor de carboidratos, e, para essas duas cultivares, observouse uma relação inversa entre teor de proteína e teor de carboidratos. Zhang e Hoseney (1998), que avaliaram farinha de milho com diferentes teores de carboidratos, concluíram que farinhas de milho com elevados teores de carboidratos apresentaram maiores valores de índice de expansão do "snack". 
TABELA 4 - Valores médios (\%) de teor de proteína, cinzas, extrato etéreo e carboidratos de grãos de milho.

\begin{tabular}{|c|c|c|c|c|}
\hline Cultivares & Proteína* & Cinzas* & Extrato etéreo* & Carboidratos* \\
\hline HD-951128 & $10,79 \mathrm{~b}$ & $1,29 \mathrm{c}$ & $4,64 \mathrm{~d}$ & $71,49 \mathrm{i}$ \\
\hline НТ-971011 & $9,15 \mathrm{~g}$ & $1,26 \mathrm{~d}$ & $4,16 \mathrm{~h}$ & $73,81 \mathrm{c}$ \\
\hline BRS 3133 & $9,56 \mathrm{f}$ & $1,32 \mathrm{~b}$ & $4,65 \mathrm{~d}$ & $72,62 \mathrm{~g}$ \\
\hline HТ-7105-3 & $10,58 \mathrm{c}$ & $1,15 \mathrm{~g}$ & $5,31 \mathrm{a}$ & $71,20 \mathrm{j}$ \\
\hline BRS 3150 & $9,86 \mathrm{e}$ & $1,30 \mathrm{c}$ & $3,90 \mathrm{k}$ & $73,17 \mathrm{e}$ \\
\hline Bozeme Amarelo & $10,20 \mathrm{~d}$ & $1,24 \mathrm{~d}$ & $4,11 \mathrm{i}$ & $72,81 \mathrm{f}$ \\
\hline BRS 2114 & $10,97 \mathrm{a}$ & $1,27 \mathrm{~d}$ & $4,61 \mathrm{~d}$ & $71,31 \mathrm{j}$ \\
\hline BRS 3101 & $9,46 \mathrm{f}$ & $1,20 \mathrm{e}$ & $4,79 \mathrm{c}$ & $72,86 \mathrm{f}$ \\
\hline BR 205 & $9,37 \mathrm{f}$ & $1,33 \mathrm{~b}$ & $4,26 \mathrm{~g}$ & $73,16 \mathrm{e}$ \\
\hline BRS 4150 & $10,22 \mathrm{~d}$ & $1,29 \mathrm{c}$ & $4,46 \mathrm{e}$ & $72,25 \mathrm{~h}$ \\
\hline BR 206 & $9,06 \mathrm{~g}$ & $1,12 \mathrm{~g}$ & $4,32 \mathrm{f}$ & $73,39 \mathrm{~d}$ \\
\hline BRS 3060 & $9,43 \mathrm{f}$ & $1,18 \mathrm{f}$ & $4,03 \mathrm{j}$ & $73,49 \mathrm{~d}$ \\
\hline BR 106 & $9,13 \mathrm{~g}$ & $1,35 \mathrm{~b}$ & $3,97 \mathrm{j}$ & $74,12 \mathrm{~b}$ \\
\hline BR 3123 & $8,22 \mathrm{~h}$ & $1,42 \mathrm{a}$ & $4,00 \mathrm{j}$ & 74,54 a \\
\hline HS-TR-3 & $11,09 \mathrm{a}$ & $1,42 \mathrm{a}$ & $5,22 \mathrm{~b}$ & $70,69 \mathrm{k}$ \\
\hline D-1000 & $9,06 \mathrm{~g}$ & $1,19 \mathrm{e}$ & $4,60 \mathrm{~d}$ & $73,48 \mathrm{~d}$ \\
\hline
\end{tabular}

*Médias seguidas de mesma letra nas colunas não diferem $(\mathbf{P} \leq \mathbf{0 , 0 5})$ pelo teste de Scott-Knott (SCOTT e KNOTT, 1974).

\section{CONCLUSÕES}

a) Endosperma: quanto ao rendimento de endosperma, as cultivares BR 106, BRS 2114, BR 3123, Bozeme Amarelo, BRS 3101, BR 206, HD951128, BRS 4150 e BR 205 destacaram-se positivamente.

b) Rendimento de grits: Os híbridos triplos BR 3123, BRS 3101 e a cultivar BR 106 foram os mais produtivos na peneira 20 mesh; portanto, os mais recomendados para a produção de grits. As cultivares BRS 2114, BR 205, BR 206, Bozeme Amarelo, BRS 3133, BRS 3060, BRS 4150, D-1000, HS-TR-3, HT-971011 apresentaram rendimento de grits intermediário, ao passo que o híbrido duplo HD-951128 foi o menos produtivo, não sendo recomendando como melhor opção econômica para utilização pela indústria de grits. c) Proteínas e Carboidratos: Os grãos da cultivar BR 3123 tiveram teor baixo de proteína, porém, alto teor de carboidratos, sendo, portanto, os mais recomendados para a produção de grits. Os grits das cultivares HT971011, BRS 3123, BRS 3101 e BR 205 tiveram baixos teores de proteínas e altos teores de carboidratos.

\section{REFERÊNCIAS BIBLIOGRÁFICAS}

ANDERSON, R. A.; CONWAY, H. F.; PFEWER, V. F.; GRIFFIN JUNIOR, L. Gelatinization of Corn Grits by Roll and Extrusion-Cooking. Cereal Science Today, Minneapolis, v. 14, n. 1, p. 4-11, 1969.

AMERICAN ASSOCIATION OF CEREAL CHEMISTS. Approved methods of the American Association of Cereal Chemists. Saint Paul, 1983. v. 1. (AACC Methods: 44-15A, 46-13, 30-25, 08-01). 
BREKKE, O. L. Corn dry-milling: pre-tempering lowmoisture corn. Cereal Chemistry, Saint Paul, v. 44, n. 5, p. 521-531, Sept./Oct. 1967.

BREKKE, O. L.; GRIFFIN JUNIOR, E. L.; BROOKS, P. Dry-milling of opaque-2 (high lysine) corn. Cereal Chemistry, Saint Paul, v. 48, n. 5, p. 499-511, Sept./Oct. 1971

FLETCHER, S. I.; RICHMOND, P.; SMITH. A. C. An experimental study of twin screw extrusion-cooking of maize grits. Journal of Food Engineering, Essex, v. 4 , p. 291-312, 1985.

MESTRES, C.; LOUIS-ALEXANDRE, A.; MATENCIO, F. Dry-milling properties of maize. Cereal Chemistry, Saint Paul, v. 68, n. 1, p. 51-56, Jan./Feb. 1991.
PEPLINSKI, A. J.; ANDERSON, R. A.; ECKHOFF, S. R. A dry-milling evaluation of trickle sulfur dioxide treated corn. Cereal Chemistry, Saint Paul, v. 61, n. 4, July/Aug. 1984.

ROKEY, G. J.; HUBER, G. R. Corn quality assurance manual. Saint Paul: [s.n.], 1992. 101 p.

SCOTT, A. J.; KNOTT, M. A cluster analysis method for grouping means in the analysis of variance. Biometrics, Washington, v. 30, n. 3, p. 507-512, sept. 1974.

ZHANG, W.; HOSENEY, R. C. Factors affecting expansion of corn meals with poor and good expansion properties. Cereal Chemistry, Saint Paul, v. 75, n. 5, p. 639-643, Sept./Oct. 1998. 